INTERNATIONAL JOURNAL OF RESEARCHES IN BIOSCIENCES, AGRICULTURE AND TECHNOLOGY (C) VISHWASHANTI MULTIPURPOSE SOCIETY (Global Peace Multipurpose Society) R. No. MH-659/13(N) www.vmsindia.org

\title{
FERRO-ELECTRICS: APPLICATIONS BULK VS NANOSCALE
}

\author{
A. N. Wazalwar \\ Dr Ambedkar College, Deekshabhoomi, Nagpur-440010, India \\ Email: aartiwazalwar@yahoo.com
}

\begin{abstract}
:
A variety of applications of bulk ferroelectrics are discussed. Further, how the applications change if the ferroelectrics are made and studied at nanoscale level are stated and discussed. Bulk Ferroelectric Materials find applications as and in Capacitors, Non-volatile memory, Piezoelectrics for ultrasound imaging and actuators, Electro-optic materials for data storage applications, Thermistors etc. As against that ferroelectric nanomaterials find their use in numerous applications like non volatile memory devices, Ferroelectric Field Effect Transistor, photovoltaics, energy cells, biomarkers, sensors, high capacity capacitors, cleaning of environment pollution. The possibility of how making ferroelectrics at nanoscale enhances its applications is discussed.
\end{abstract}

\section{Introduction}

Ferroelectrics are dielectrics crystals which a spontaneous electric polarization due to atomic displacement in the crystal structure. The direction of polarization can be reoriented by an external electric field. Ferroelectrics also possess domains where the spontaneous polarization is parallel and is characterized by the hysteresis loop.

'Nanoscience is the study of phenomena and manipulation of materials at atomic, molecular and macromolecular scales, where properties differ significantly from those at a larger scale'. Bulk materials (the 'big' pieces of materials we see around us) possess continuous (macroscopic) physical properties. The same applies to micron-sized materials (e.g. a grain of sand). But when particles assume nanoscale dimensions, the principles of classic physics are no longer capable of describing their behaviour (movement, energy, etc.): at these dimensions, the principles of quantum mechanics principles. The same material (e.g. gold) at the nanoscale can have properties (e.g. optical, mechanical and electrical) which are very different from (and even opposite to!) the properties the material has at the macroscale (bulk).

We feel this work is particularly important: in fact, nanosciences and nanotechnologies are at the forefront of today's science and technology, engineering both matter and living systems at the scale of molecules and atoms. Their unique applications, products, markets and profitable revenue sources can bring new benefits and challenges to both society and economy, as they are becoming increasingly embedded in today's life. In fact, society at large needs to understand their pros and cons to make responsible choices about new products and processes: research, innovation and education constitute a living ecosystem, where education plays a key role.

First, at the nanometre scale, the properties of matter, such as energy, change. This is a direct consequence of the small size of nanomaterials, physically explained as quantum effects. The consequence is that a material (e.g. a metal) when in a nano-sized form can assume properties which are very different from those when the same material is in a bulk form. For instance, bulk silver is non-toxic, whereas silver nanoparticles are capable of killing viruses upon contact. Properties like electrical conductivity, colour, strength and weight change when the nanoscale level is reached: the same metal can become a semiconductor or an insulator at the nanoscale level.

Ferroelectric materials exhibit a wide spectrum of functional properties, including switchable polarization, piezoelectricity, high non-linear optical activity, pyroelectricity, and non-linear dielectric behaviour. These properties are indispensable for application in electronic devices such as sensors, microactuators, infrared detectors, microwave phase filters and, ultimately, non-volatile memories[1-9]. They are technologically important materials, particularly for their applications in ferroelectric random access memory, based on semiconductor integrated technology have been a great success. Furthermore, these RAMS are very sensitive to radiation and this is detrimental for military and space applications.

Applications of Ferroelectric Materials
$\begin{aligned} & \text { - } \\ & \text { - } \\ & \text { - } \\ & \text { and actuaton-volatile memory } \\ & \text { Piezoelectrics for ultrasound imaging }\end{aligned}$


- Electro-optic materials for data storage applications

- Thermistors

- Switches known as transchargers or transpolarizers

- Oscillators and filters

- Light deflectors, modulators and displays

Among the important ferroelectric materials lead zirconate titanate (PZT), which is part of the solid solution formed between ferroelectric lead titanate and anti-ferroelectric lead zirconate with different compositions are used for different applications. Recent works indicate the influence of nanoparticles in PZT properties like decrease of synthesizing temperature, electrical conductivity and low dielectric loss. These materials will be the future ferroelectric materials for novel applications.

Recent advances in fabrication and characterization of oxide ferroelectrics present both opportunities and challenges. Nanoscale ferroelectric structures have a high potential for application in electronic devices, such as high-density non-volatile memories.

Scaling of the device dimensions to the range where ferroelectric materials start to show a pronounced size effect has emphasized the importance of nanoscale studies of ferroelectric properties. The size effect in ferroelectrics manifests itself with the reduction in the sample geometrical dimensions by a decrease in the remnant polarization, dielectric permittivity and phase transition temperature, increase in the coercive field, changes in the domain structure, etc [10].

Device miniaturization poses not only technological and manufacturing challenges, it also requires the understanding of the phenomena occurring at nanoscale in condensed matter and at the involved interfaces. Ferroelectric (FE) materials, such as the renowned perovskites _BaTiO3, $\mathrm{PbZr} x \mathrm{Ti} 1-x \mathrm{O} 3, \quad \mathrm{BiFeO} 3$ and layered perovskites _SrBi2Ta2O9 _Bi,La_4Ti3O12_, have been proposed for nonvolatile ferroelectric random access memories since two decades. Ferroelectric perovskites can hold information in the form of the polarization direction of individual $\mathrm{FE}$ domains, because they usually have two thermodynamically equivalent ground states of opposite ionic polarization.

In the past decades, ferroelectric materials have attracted wide attention due to their applications in nonvolatile memory devices (NVMDs) rendered by the electrically switchable spontaneous polarizations. Furthermore, the combination of ferroelectric and nanomaterials opens a new route to fabricating a nanoscale memory device with ultrahigh memory integration, which greatly eases the ever increasing scaling and economic challenges encountered in the traditional semiconductor industry. FeFET (Ferroelectric Field Effect Transistors at nanoscale are being hence integrated.

The enormously broad range of materials exhibiting ferroelectricity and the variety of their physical properties result in numerous applications of bulk ferroelectrics [4]. Table 1 shows the connections between different physical effects exhibited by bulk ferroelectrics and their applications. Recent advances in nanotechnologies, especially in nanoinstrumentation (for example, scanning probe microscopy [5]) and materials nanofabrication [6] allowed the direct probing of ferroelectricity at the nanoscale. The new and unexplored world of nanoscale ferroelectrics (nanoparticles of different shapes and sizes, nanofilms, nanopatterned structures, etc.) raised fundamental questions and stimulated very active research in both academic and industrial sectors [7]. As a result, a new era of nanoscale ferroelectrics was launched.

Novel effects, associated with reduced dimensions and found in nanoscale ferroelectrics, highlighted exciting possibilities for new applications reviewed recently in [8]. Almost all of the attention for the mentioned review [8] was devoted to the thin film nanoscale device structures (which can be easily integrated with a Si chip) with focus on ultrafast switching, electrocaloric coolers for computers, phase-array radar, threedimensional trenched capacitors for dynamic random access memories, room temperature magnetic field detectors, and miniature X-ray and neutron sources. So far, we have not found a coherent review summarizing the actual and possible applications of ferroelectric nanoparticles.

\section{Physical effect/property Applications}

Ferroelectric hysteresis : Nonvolatile computer information storage

High relative permittivities (several thousands) : Capacitors

Direct piezoelectric effect : Sensors (microphones, accelerometers, hydrophones, etc.) 
Converse piezoelectric effect : Actuators, ultrasonic generators, resonators, filters Pyroelectric effect : Uncooled infra-red detectors

Electro-optic effects : Laser Q-switches, optical shutters and integrated optical (photonic) devices

Nonlinear optical effects : Laser frequency doubling, optical mixing, including four-wave mixing and holographic information storage

Coupling between stress and birefringence : Radar signal processing

Positive temperature coefficient of resistance (PTCR): Electric-motor overload- protection devices and self-stabilizing ceramic heating elements

\section{Applications of bulk ferroelectrics Applications of Ferroelectric materials (Recent Applications)}

In recent years, ferroelectric materials and thin films have attracted much attention and exhibited potential in many important applications such as

- dynamic random access memories (DRAMS),

- non-volatile ferroelectric random access memories micro-armours and

- infrared sensors.

In order to explore novel physical properties and phenomena and realize potential applications of nanostructures and nanomaterials, the ability to fabricate and process nanomaterials and nanostructures is the first corner stone in nanotechnology.

Multifunctional ferroelectric materials offer a wide range of useful properties, from switchable polarization that can be applied in memory devices to piezoelectric and pyroelectric properties used in actuators, transducers and thermal sensors. At the nanometer scale, however, material properties are expected to be different from those in bulk. Fundamental problems such as the superparaelectric limit, the influence of the free surface, and of interfacial and bulk defects on ferroelectric switching, etc., arise when scaling down ferroelectrics to nanometer sizes. In order to study these size effects, fabrication methods of high quality nanoscale ferroelectric crystals have to be developed.

At present, the ferroelectric materials suitable for these devices are $\mathrm{Pb}(\mathrm{Zr}, \mathrm{Ti}) \mathrm{O}_{3}(\mathrm{PZT})$ systems, $\mathrm{SrBi}_{2} \mathrm{Ta}_{2} \mathrm{O}_{9}(\mathrm{SBT}) \quad$ systems, $\mathrm{Bi}_{4} \mathrm{Ti}_{3} \mathrm{O}_{12}(\mathrm{BIT})$ systems and $\mathrm{BaTiO}_{3}(\mathrm{BT})$ systems that are studied with a great deal of interest. In these ferroelectric materials, BaTio.91 $\left(\mathrm{Hf}_{0.5}, \mathrm{Zr}_{0.5}\right)_{0.09} \mathrm{O}_{3}$ (BTHZ-9), one of the
BT systems, which has several advantages such as

- an extremely low coercive field,

- a high remnant polarization,

- better mechanical strength and

- small deviation in composition, could have a strong potential application for ferroelectric thin film devices.

In addition, the BTHZ systems, lead and/or bismuth-free material, present a great interest both for applications in the field of environmental protection and for fundamental studies.

\section{Applications as and in nanostructures}

In the past four years in several countries, including Russia, Germany, the UK, and the USA, ferroelectric nano-structures have been produced and characterized. Materials with small dimensions like nanomaterials are very important because their properties differ significantly from those of their bulk counterparts. Nanomaterials are widely used in various applications such as photovoltaics, energy cells, biomarkers, sensors, high capacity capacitors, cleaning of environment pollution and in many others. The ferroelectric effect is an electrical phenomenon whereby certain crystals may exhibit a spontaneous dipole moment (which is called ferroelectric by analogy with ferromagnetic - exhibiting a permanent magnetic moment).

\section{FERROELECTRIC NANOWIRES, AND NANODOTS Devices}

At present three groups are working on prototype self-trenched capacitors for DRAMs or FRAMs. The most advanced of these is a cooperative project involving IMEC in Belgium, S T Microelectronics in France, and groups at the University of Toulon. They have produced [13,14] a mushroom-shaped FRAM structure with the top electrode and an SBT capacitor extending down the sidewall. This device gives a $50 \%$ increase in capacitance for the same area on the chip and is nearly ready to manufacture.

Another successful step towards [3D] nano-ferroelectric devices is trenched electroding. This one is from a Samsung-Tokyo Institute of Technology collaboration of Funakubo et al [15]. They achieve excellent step coverage using CVD ruthenium from an organic ruthenium-DER compound (Tosoh Corp.).

\section{PVDF devices}


Private communication to the authors from Intel Corp. reveals that they have had an active program to make digital computer FRAM memories from PVDF films.

PZT

Lead zirconate titanate is one of two ferroelectrics used for FRAMs and is favored by Samsung, Toshiba, Fujitsu, and others (with IMEC, ST Microelectronics, and Matsushita/Panasonic favoring SBT). Several device issues remain.

\section{$\mathrm{KNO}_{3}$}

Although recently neglected for device purposes because of its extreme water solubility, potassium nitrate retains great fundamental interest as an easily-fabricated thin-film ferroelectric. In bulk its ferroelectric phase is stable over a very narrow temperature range and is reentrant, accessible only upon cooling, but in thin-film form the ferroelectric phase temperature width varies as reciprocal thickness $1 / \mathrm{d}$ and can exceed $200 \mathrm{~K}$, including the full military and commercial operating range $(-50$ to $+130 \mathrm{~F})$.

\section{ULTRA-THIN SINGLE CRYSTALS}

Two systems have been fabricated via focussed ion beam (FIB) techniques down to ca. $70 \mathrm{~nm}$ thickness by Gregg et al. In Belfast. The first is BaTiO3 - for which the SEM results of nanodomain structures are amazing. Faster, lighter computers possible with nanotechnology, Smaller, lighter computers and an end to worries about electrical failures sending hours of on-screen work into an inaccessible limbo mark the potential result of Argonne research on nano ferroelectric crystals.

Argonne researchers have learned that they are more chemically reactive, exhibit new electronic properties and can be used to create materials that are stronger, tougher and more resistant to friction and wear than bulk materials.

Improved nano-engineered ferroelectric crystals could realize a 50-yearold dream of creating nonvolatile random access memory (NVRAM). The first fruits of it can be seen in Sony's PlayStation 2 and in smart cards now in use in Brazil, China and Japan. A simple wave of a smart card identifies personnel or pays for gas or public transportation.

\section{Nanoferroelectric}

RAM - random access memory is used when someone enters information or gives a command to the computer. It can be written to as well as read but - with standard commercial technology - holds its content only while powered by electricity. Argonne materials scientists have created and are studying nanoscale crystals of ferroelectric materials that can be altered by an electrical field and retain any changes.

Because the crystals do not revert spontaneously, RAM made with them would not be erased should there be a power failure. Laptop computers would no longer need backup batteries, permitting them to be made still smaller and lighter. There would be a similar impact on cell phones. Achieving such permanence is a long-standing dream of the computer industry.

"Companies such as AT\&T, Ford, IBM, RCA and Westinghouse Electric made serious efforts to develop non-volatile RAMs in the 1950s, but couldn't achieve commercial use," said Argonne researcher Orlando Auciello.

"Working on the nanoscale changes this," said Auciello. "It means higher density memories with faster speeds and megabyte (the amount of memory needed to store one million characters of information) - or even gigabyte (one billion bytes) - capacity. Nanoferroelectric Smart cards don't forget. Argonne scientists are using their expertise in ferroelectrics to improve smart cards. These are the size and shape of credit cards but contain ferroelectric memory that can carry substantial information, such as its bearer's medical history for use by doctors, pharmacists and even paramedics in an emergency. Unlike magnetic strips on credit cards, these memories do not come in contact with their readers and will not wear out.

Current smart cards carry about 250 kilobytes of memory. Argonne researchers are collaborating with the Colorado Springs, Colo., Symetrix Corp. to develop a higher capacity card with a more flexible and longer-lasting memory.

Nanomaterials have been studied at Argonne since the 1980s. They are now one of the hottest research topics worldwide. Several nanoscale materials research centers are being planned by the U.S. Department of Energy (DOE), with one likely to be built at Argonne.

"Nanoscale ferroelectrics allow us to develop better multilayer capacitors, which could be used in even smaller cell phones," added Stephen Streiffer, a colleague of Auciello's. He also sees application in motors to power micro- and nano-electro-mechanical systems. 
"Nature likes to put things together in certain ways," Streiffer said. "As we learn more about nanoscience - when we can control construction at the nanoscale - we will be able to engineer the nanoworld differently and create novel combinations. We should find new materials, things we can't even imagine yet."

Argonne's broad program in ferroelectrics includes making and studying the properties of ferroelectrics using a time-of-flight ion scattering and recoil spectroscopy system developed by Auciello and Argonne colleagues A.R. Krauss and D.M. Gruen in conjunction with J.A. Schultz of Ionwerks." Ferroelectrics, he said, are also investigated using state-ofthe-art in-situ X-ray scattering techniques at Argonne's Advanced Photon Source, the nation's brightest source of hard X-rays for materials research, by a Materials Science Division team consisting of G.B. Stephenson, J.A. Eastman, C. Thompson (Northern Illinois / Argonne), Streiffer, and Auciello.

\section{Conclusions}

Nano-ferroelectrics are of considerable interest for commercial devices where lateral area must now be $<0.2 \mu \mathrm{m} 2$ and thicknesses ca. $120 \mathrm{~nm}$ for FRAMs and thinner for DRAMs. Scientists are rapidly acquiring an understanding of what physical effects occur at these dimensions, and which are extrinsic and which intrinsic.

\section{References}

[1] Szafraniak, C. Harnagea, R. Scholz, S. Bhattacharyya, D. Hesse, and M. Alexe 83,11, 2003, 2211-2213

[2] Ionela Vrejoiu,a_ Marin Alexe, Dietrich Hesse, and Ulrich Gosele Ferroelectric nanostructures J. Vac. Sci. Technol. B 27„1 Jan/Feb 2009 pp. 498-503.
[3] A Gruverman and A Kholkin Nanoscale ferroelectrics: processing, characterization and future trends Rep. Prog. Phys. 69 (2006) 24432474

[4] Xingqiang Liu , Yueli Liu , Wen Chen , Jinchai Li and Ferroelectric memory based on nanostructures Nanoscale Research Letters 2012, 7:285-290

[5] Zhenkui Shen, Zhihui Chen, Qian Lu, Zhijun Qiu, Anquan Jiang, Xinping Qu, Yifang Chen and Ran Liu , Nano-embossing technology on ferroelectric thin film $\mathrm{Pb}(\mathrm{ZrO} 0.3$,Ti0.7)O3 for multi-bit storage application Nanoscale Research Letters 2011, 6:474-479,

[6] Zhaohui Ren, Gang Xu, Xiao Wei, Yong Liu, Ge Shen, and Gaorong HanwShape Evolution of $\mathrm{Pb} \quad(\mathrm{Zr}, \mathrm{Ti}) \mathrm{O} 3 \quad$ Nanocrystals Under Hydrothermal Conditions J. Am. Ceram. Soc., 90 [8] 2645-2648 (2007)

[7] Waser R (ed) 2005 Nanoelectronics and InformationTechnology: Advanced Electronic Materials and Novel Devices (Berlin: Wiley$\mathrm{VCH})$

[8] Hong S 2003 Nanoscale Phenomena in Ferroelectric ThinFilms (Dordrecht: Kluwer)

[9] Alexe M and Gruverman A 2004 Nanoscale Characterization of Ferroelectric: Scanning Probe Microscopy Approach (Berlin: Springer).

[10] Kurapati Srinivas, American Journal of Nanoscience and Nanotechnology,2014; 2(3): 662

[11] http://www.eurekalert.org/ Published online June 20, 2014 (http://www.sciencepublishinggroup.com/j/n ano)

doi: $10.11648 /$ j.nano.20140203.15 